\title{
Supporting information for \\ Photochemical grafting of n-alkenes onto carbon surfaces: the role of photoelectron ejection
}

Paula E. Colavita, Bin Sun, Kiu-Yuen Tse and Robert J. Hamers.

Department of Chemistry, 1101 University Avenue, University of Wisconsin. Madison, Wisconsin 53706-1396.

Corresponding author: rjhamers@wisc.edu

\section{Complete citation for Ref. 17 in the main text:}

Gaussian 03, Revision C.02, Frisch, M. J.; Trucks, G. W.; Schlegel, H. B.; Scuseria, G. E.; Robb, M. A.; Cheeseman, J. R.; Montgomery, Jr., J. A.; Vreven, T.; Kudin, K. N.; Burant, J. C.; Millam, J. M.; Iyengar, S. S.; Tomasi, J.; Barone, V.; Mennucci, B.; Cossi, M.;

Scalmani, G.; Rega, N.; Petersson, G. A.; Nakatsuji, H.; Hada, M.; Ehara, M.; Toyota, K.; Fukuda, R.; Hasegawa, J.; Ishida, M.; Nakajima, T.; Honda, Y.; Kitao, O.; Nakai, H.; Klene, M.; Li, X.; Knox, J. E.; Hratchian, H. P.; Cross, J. B.; Bakken, V.; Adamo, C.; Jaramillo, J.; Gomperts, R.; Stratmann, R. E.; Yazyev, O.; Austin, A. J.; Cammi, R.; Pomelli, C.; Ochterski, J. W.; Ayala, P. Y.; Morokuma, K.; Voth, G. A.; Salvador, P.; Dannenberg, J. J.; Zakrzewski, V. G.; Dapprich, S.; Daniels, A. D.; Strain, M. C.; Farkas, O.; Malick, D. K.; Rabuck, A. D.; Raghavachari, K.; Foresman, J. B.; Ortiz, J. V.; Cui, Q.; Baboul, A. G.; Clifford, S.; Cioslowski, J.; Stefanov, B. B.; Liu, G.; Liashenko, A.; Piskorz, P.; Komaromi, I.; Martin, R. L.; Fox, D. J.; Keith, T.; Al-Laham, M. A.; Peng, C. Y.; Nanayakkara, A.; Challacombe, M.; Gill, P. M. W.; Johnson, B.; Chen, W.; Wong, M. W.; Gonzalez, C.; and Pople, J. A.; Gaussian, Inc., Wallingford CT, 2004.

\section{Covalent functionalization of $\mathrm{H}$-terminated a-C: dark control experiments}

Figure 1(a) and 1(b) show the results of control experiments aimed at testing if $254 \mathrm{~nm}$ light was necessary to functionalize $\mathrm{H}$-terminated amorphous carbon. Figure 1(a) shows the XPS profiles of two amorphous carbon samples that had been H-terminated and then covered by a thin film of liquid TFAAD for $15 \mathrm{~h}$ under inert atmosphere. The top curve shows the results obtained for a typical sample exposed to $254 \mathrm{~nm}$ light; the $\mathrm{F}(1 \mathrm{~s}), \mathrm{O}(1 \mathrm{~s})$ 

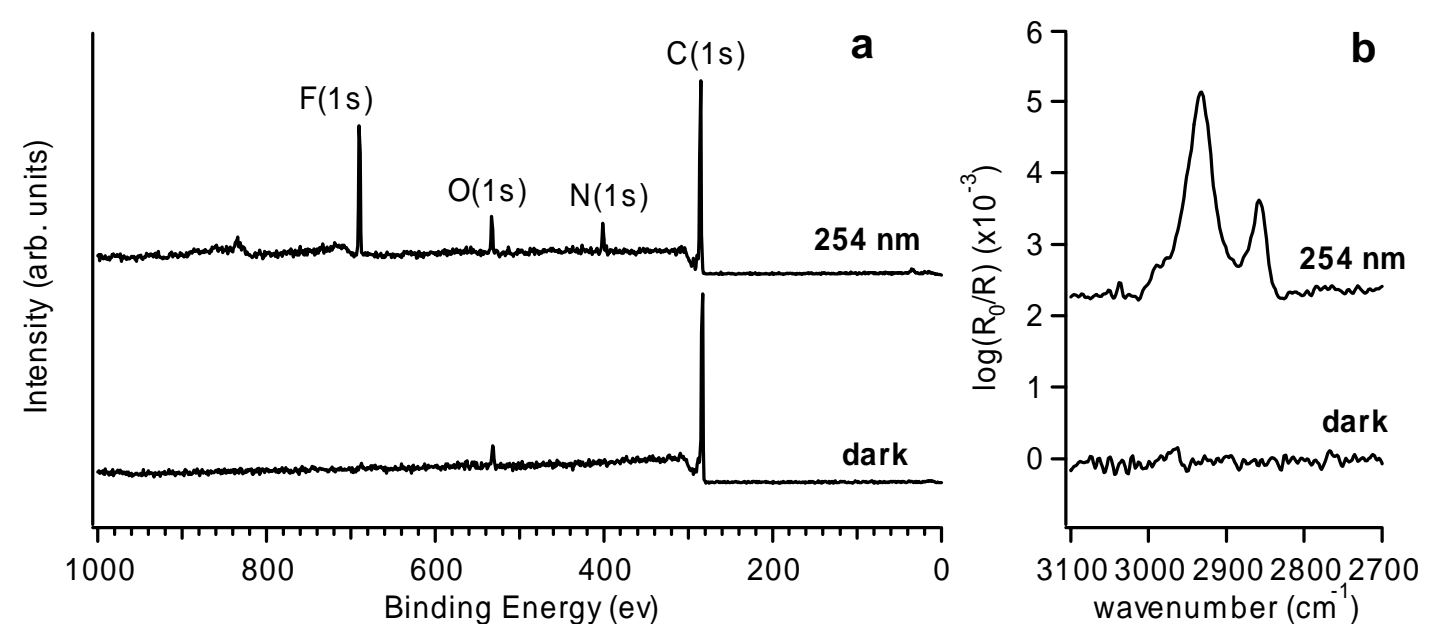

Figure 1: Dark controls for the covalent functionalization of $\mathrm{H}$-terminated amorphous carbon with TFAAD. XPS survey scans (a) and IRRAS spectra in the $\mathrm{CH}_{2}$ stretching region after $15 \mathrm{~h}$ under $254 \mathrm{~nm}$ illumination and in the dark. There is no evidence of reaction in the ahsence of illumination.

and N(1s) peaks are clearly visible in the survey spectra. The bottom curve shows the results obtained when a sample was kept in the dark for the same length of time; the $\mathrm{F}(1 \mathrm{~s})$ signal is barely visible in this case. Fitting of the $\mathrm{F}(1 \mathrm{~s})$ and $\mathrm{C}(1 \mathrm{~s})$ regions of this sample revealed that the $A_{\mathrm{F}(1 \mathrm{~s})} / A_{\mathrm{C}(1 \mathrm{~s})}$ ratio is 0.011 , in contrast with a typical ratio of $0.203 \pm$ 0.010 obtained after $15 \mathrm{~h}$ illumination. The $A_{\mathrm{O}(1 \mathrm{~s})} / A_{\mathrm{C}(1 \mathrm{~s})}$ ratio was found to be 0.053 which is the typical value observed for bare H-terminated amorphous carbon (see text).

Figure 1(b) shows spectra obtained for TFAAD on H-terminated carbon after $15 \mathrm{~h}$ illumination (top trace) and after $15 \mathrm{~h}$ in the dark (bottom trace) in the $\mathrm{CH}_{2}$ stretching region. No peaks are visible for the sample that was kept in the dark, suggesting that no reaction took place in the absence of illumination. Although a small amount of physisorbed molecules might be present, as suggested by the XPS F(1s) residual signal, their surface concentration remains below the detection level of our IRRAS technique. 


\section{Reference FTIR spectra of the four alkenes}

Figure 2 shows the spectra of the four alkenes used for our experiments, measured in a solution of carbon tetrachloride. The four alkenes have clearly distinguishable spectral profiles. Common to all of the compounds are the $\mathrm{C}=\mathrm{C}$ stretching of the terminal olefin group $\left(\sim 1640 \mathrm{~cm}^{-1}\right)$ and the vinyl C-H stretchings $\left(\sim 2980, \mathrm{~cm}^{-1}\right)$. Peak positions and assignments have been discussed on previous work and are summarized on Table 1.

\begin{tabular}{|c|c|c|c|c|}
\hline \multicolumn{5}{|c|}{ Peak positions $\left(\mathrm{cm}^{-1}\right)$} \\
\hline Assignments $^{a}$ & TFAAD $^{2}$ & dodecene & $\mathrm{tBOC}^{3}$ & $\mathbf{U A}-\mathbf{M e}^{3}$ \\
\hline$v(\mathrm{~N}-\mathrm{H})$ & 3446 & - & 3462 & $\overline{--}$ \\
\hline$v\left(\mathrm{H}_{2} \mathrm{C}==\right) \mathrm{s}$ & $\begin{array}{c}3080 \\
3066(\mathrm{sh}) \\
2996(\mathrm{sh})\end{array}$ & $\begin{array}{c}3079 \\
3066 \\
2995(\mathrm{sh})\end{array}$ & $\begin{array}{c}3078 \\
3063 \\
3001(\mathrm{sh})\end{array}$ & $\begin{array}{c}3079 \\
3067 \\
3019 \text { (sh) }\end{array}$ \\
\hline $\mathrm{v}\left(\mathrm{CH}_{3}\right) \mathrm{a}$ & - & 2958 & 2979 & 2952 \\
\hline $\mathrm{v}\left(\mathrm{CH}_{2}\right) \mathrm{a}$ & 2930 & 2927 & 2930 & 2930 \\
\hline $\mathrm{v}\left(\mathrm{CH}_{2}\right) \mathrm{s}$ & 2858 & 2856 & 2856 & 2857 \\
\hline$v(\mathrm{C}==\mathrm{O})$ & 1703 & - & 1721 & 1743 \\
\hline$v(C==C)$ & 1642 & 1642 & 1641 & 1641 \\
\hline $\begin{array}{l}\beta(\mathrm{NH}) v(\mathrm{CN}) \\
v(\mathrm{CC})\end{array}$ & 1561 & - & 1504 & - \\
\hline$\beta\left(\mathrm{CH}_{2}\right)$ & 1467 & 1465 & 1460 & 1460 \\
\hline$\beta\left(\mathrm{CH}_{3}\right)$ & - & 1379 & $\begin{array}{l}1392 \\
1367\end{array}$ & 1436 \\
\hline$v(\mathrm{C}-\mathrm{O})$ & - & - & 1173 & $\begin{array}{l}1198 \\
1172\end{array}$ \\
\hline$v(\mathrm{C}-\mathrm{F})$ & $\begin{array}{l}1210 \\
1185 \\
1168\end{array}$ & - & - & - \\
\hline
\end{tabular}

Table 1: The parent compounds were dissolved in carbon tetrachloride and the spectra were taken at $1 \mathrm{~cm}^{-1}$ resolution. The spectra of organic layers were obtained at $4 \mathrm{~cm}^{-1}$ resolution with p-polarized light at $80^{\circ}$ incidence. The $n$-alkene peak assignments are based on previous work from our laboratory ${ }^{2,3}$ except for dodecene, whose peaks were interpreted on the basis of assignments reported for the spectrum of bulk and monolayer alkanethiols. $^{4,5}$

$a \mathrm{v}=$ stretching; $\beta=$ bending; $\mathrm{a}=$ asymmetric $; \mathrm{s}=$ symmetric. 


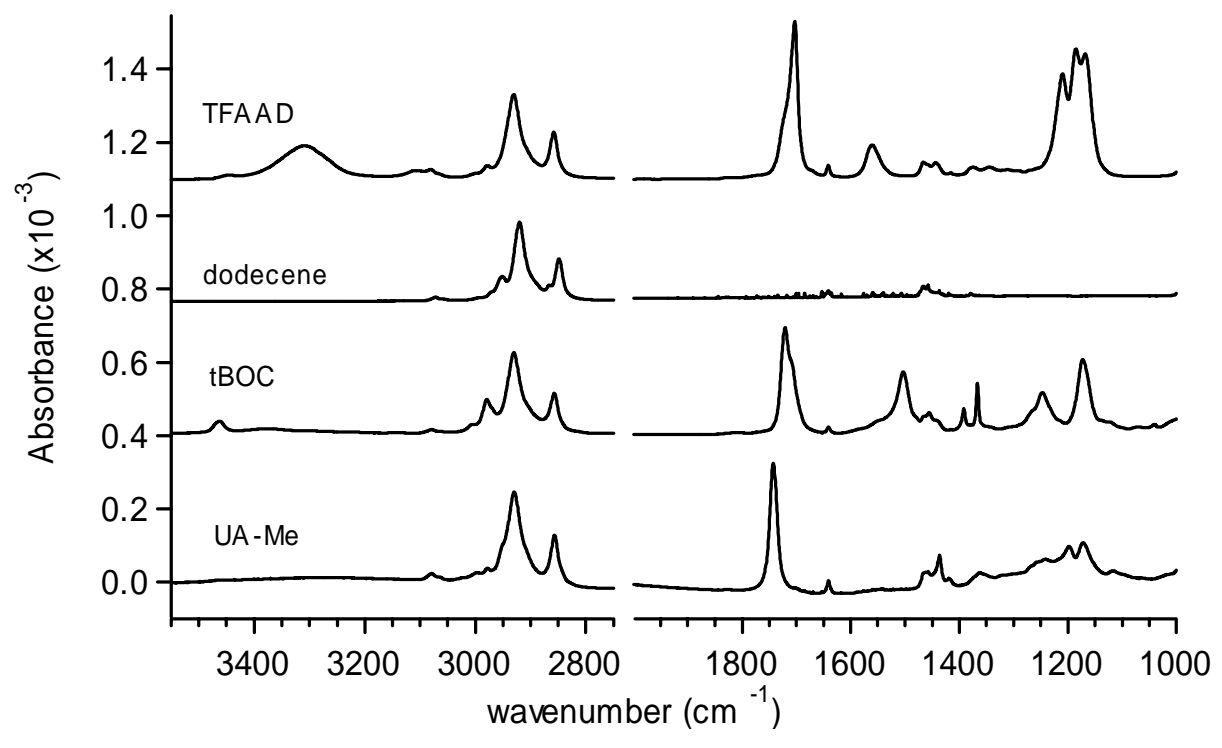

Figure 2: Reference spectra measured in solution of the four alkenes used in this study. The spectra were baseline corrected and normalized to facilitate comparison. Peak positions and assignments are reported on Table 1. 


\section{XPS and UPS of as-deposited carbon}

Figure 3 shows the XPS spectra of the $\mathrm{C}(1 \mathrm{~s})$ and $\mathrm{O}(1 \mathrm{~s})$ regions of the a-C film as deposited and after hydrogen-termination. The only elements present in the XPS survey scans were oxygen and carbon. Nitrogen, which is known to strongly influence the electronic properties of amorphous carbon materials, was never observed in the XPS spectra as long as the sputtering base pressure was kept at or below $2 \times 10^{-6}$ Torr. The $\mathrm{C}(1 \mathrm{~s})$ spectrum of the as deposited sample (A) shows an asymmetric broad line characteristic of amorphous carbon. ${ }^{6,7}$ The spectrum was fitted with four components, centered at 284.1, 284.8, 286.0 and $288.0 \mathrm{eV}$. The first two peaks are separated by 0.7 $\mathrm{eV}$, which is consistent with the separation between $\mathrm{sp}^{2}$ and $\mathrm{sp}^{3}$ carbon in disordered graphite and other amorphous carbon samples. ${ }^{6,7}$ However, the intensities of these two components are highly correlated in the fit landscape, and determining the percent composition of these two phases is challenging. The component at $286.0 \mathrm{eV}$ has previously been attributed to $\pi-\pi^{*}$ transitions, which are thought to appear in fullerenes and disordered systems at much lower energies above the main line than in HOPG. ${ }^{7,8}$ This feature is broader than the 1.1-1.3 eV FWHM expected from the spectral resolution, and it likely also comprises high binding energy carbon. Oxygen was also found in the XPS spectra of the as deposited samples and we attribute part of this peak, as well as the component at $288.0 \mathrm{eV}$, to oxidized forms of carbon. This particular as-deposited sample yielded a ratio of $\mathrm{O}(1 \mathrm{~s})$ and $\mathrm{C}(1 \mathrm{~s})$ peak areas of 0.139 (already corrected by the sensitivity factors). The amount of oxygen was found to vary in the range $0.090-0.170$; this variation in the oxygen content could be due partly to the presence of adsorbed water on the samples.

Figure 4 shows the He I UPS spectrum of the as-deposited sample. Three broad features can be observed in the spectral profile at approximately 4.8, 7.0 and 9.7 eV. Previous studies on oxidized pyrocarbon and carbon blacks have shown that $\mathrm{O} 2 p$ states of oxygen species in the -1 oxidation state $\left(\mathrm{O}_{2}^{-}\right.$or $\left.\mathrm{O}^{-}\right)$as well as carbonates yield peaks in the range 4.6-4.8 eV; the shoulder we observe at $4.8 \mathrm{eV}$ could therefore be assigned to such species. The features at 7.0 and $9.7 \mathrm{eV}$ can be assigned to molecular states of adsorbed water, which have been observed at 7.0 and $9.7 \mathrm{eV}$ on oxidized grafoil and pyrocarbon, ${ }^{9}$ or to $\mathrm{OH}^{-}$groups, previously observed at 6.9 and $10.5 \mathrm{eV}$ on glassy 
carbon. ${ }^{10}$ Upon hydrogen-termination the $\mathrm{O} 2 p$ features at 4.8 and $9.8 \mathrm{eV}$ are greatly reduced, in agreement with the loss of oxidized species observed in the XPS spectra.

To measure the work function of each sample, the sharp cutoff in electron emission at high binding energy was fit to a line and extrapolated to find the high binding-energy cutoff; this value was then subtracted from the incident photon energy $(21.2 \mathrm{eV})$ to yield the work function. ${ }^{11,12}$ This procedure resulted in a work function of $4.8 \mathrm{eV}$ for the asdeposited sample.

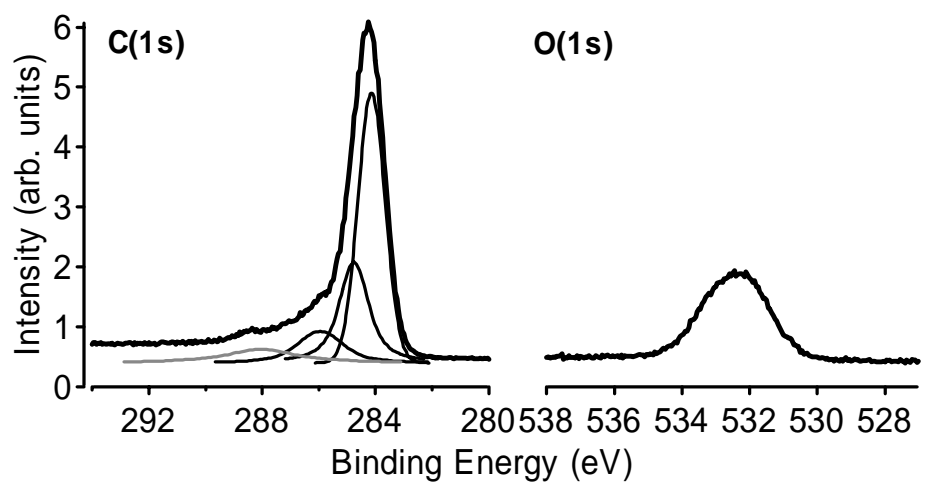

Figure 3: XPS in the $\mathrm{C}(1 \mathrm{~s})$ and $\mathrm{O}(1 \mathrm{~s})$ regions of the as-deposited sample. The individual contributions to the $\mathrm{C}(1 \mathrm{~s})$ peak were obtained via fitting procedures and are reported under the $\mathrm{C}(1 \mathrm{~s})$ peak.
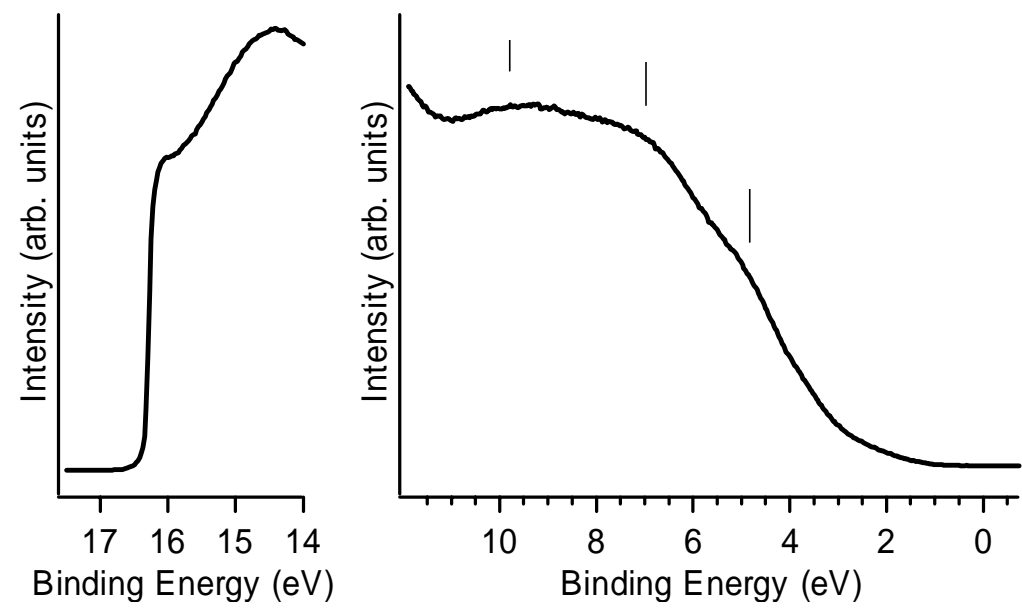

Figure 4: He I UPS spectra of the as deposited sample. The broad features at 4.8, 7.0 and $9.7 \mathrm{eV}$ are indicated with lines on the graph. 


\section{Impedance spectroscopy of H-terminated amorphous carbon}

Figures 5( $\mathrm{a}$ and $\mathrm{b}$ ) show the area normalized impedance spectra of the hydrogen terminated sample for three different concentrations of $\mathrm{KCl}$ electrolyte: $0.01,0.10$ and 1.0 M. The impedance spectra were collected by applying a sinusoidal potential of $10 \mathrm{mV}$ amplitude centered at the open circuit potential, found at $0.40,0.36$ and $0.28 \mathrm{~V}$ (vs. $\mathrm{Ag} / \mathrm{AgCl} / 3 \mathrm{M} \mathrm{KCl}$ ) for $0.01,0.1$ and $1 \mathrm{M}$ solutions respectively, while scanning over a wide frequency range $\left(0.1\right.$ to $\left.10^{5} \mathrm{~Hz}\right)$. The in-phase and out-of-phase current response with respect to the excitation $\mathrm{AC}$ voltage were collected and the impedance was expressed as a frequency dependent complex quantity ${ }^{\wedge} Z={ }^{\wedge} \mathrm{V} /{ }^{\wedge} \mathrm{I}=\mathrm{Z}$ ' $+\mathrm{i} Z$ ”; the plots show the magnitude $|\mathrm{Z}|$ and phase angle $(\theta)$ of the impedance spectrum.

The three impedance spectra show similar behavior. At high frequencies the impedances level off, becoming frequency-independent; this indicates that beyond a certain threshold frequency the response is essentially resistive, with the resistance determined by the electrolyte concentration. At low frequencies the phase angle of $-82^{\circ}$ is close to that expected of an ideal capacitor $\left(-90^{\circ}\right)$, indicating that the interface can be approximated by an ideal capacitor. The deviation from $-90^{\circ}$ can be explained by the presence of conductive defects at the interface, so that the interface is more accurately described by a capacitor in parallel to a large but finite resistance.

In order to obtain an estimate of the interfacial capacitance, the impedance data were fitted according to the Randles model ${ }^{13}$

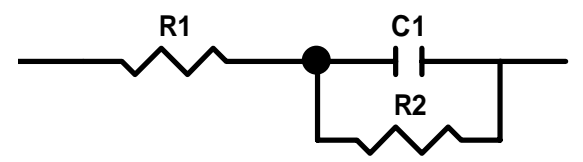

where $\mathrm{R} 1$ is the resistance of the $\mathrm{KCl}$ solution, and $\mathrm{C} 1$ and $\mathrm{R} 2$ are the capacitance and resistance associated to the interface. The capacitance values thus calculated were 5.4, 4.8 and $5.0 \mu \mathrm{F} / \mathrm{cm}^{2}$ for the $0.01,0.1$ and $1.0 \mathrm{M} \mathrm{KCl}$ concentrations respectively. The capacitance is insensitive to the $\mathrm{KCl}$ concentration used in the measurements, in contrast to what would be expected from capacitances associated to the interfacial double-layer. In fact, according to Gouy-Chapman theory the double-layer capacitance is proportional to the square root of the ion concentration; ${ }^{14}$ for the concentrations used in these experiments we would therefore expect an increase in the capacitance by a factor of 10 
when the concentration is increased from 0.01 to $1.0 \mathrm{M}$. Furthermore, double-layer capacitances in $0.1 \mathrm{M}$ electrolyte solutions are typically an order of magnitude larger than the $5 \mu \mathrm{F} / \mathrm{cm}^{2}$ value observed in our experiments. ${ }^{14}$ These results suggest that a capacitance smaller and in series to that of the double-layer dominates the impedance response at low frequencies; and that this capacitance is essentially determined by the electronic properties of the electrode rather than by the electrolyte concentration. The origin of this capacitance is likely a space-charge layer at the carbon electrode surface, as discussed in the text.

The Mott-Schottky plots presented in the text, indicate that hydrogen-terminated electrodes are in depletion at the potential at which the impedance data of Figures 5(a) and (b) was collected. This result further confirms that the capacitance at the open circuit potential of the above described impedance measurements is dominated by a space charge layer. Figure 5(c) shows the capacitance vs. polarization plot obtained at $10 \mathrm{~Hz}$ in 0.01 and $0.1 \mathrm{M} \mathrm{KCl}$ obtained by modeling the impedance data as a series RC circuit, and used to generate the Mott-Schottky plot reported in the main text. 

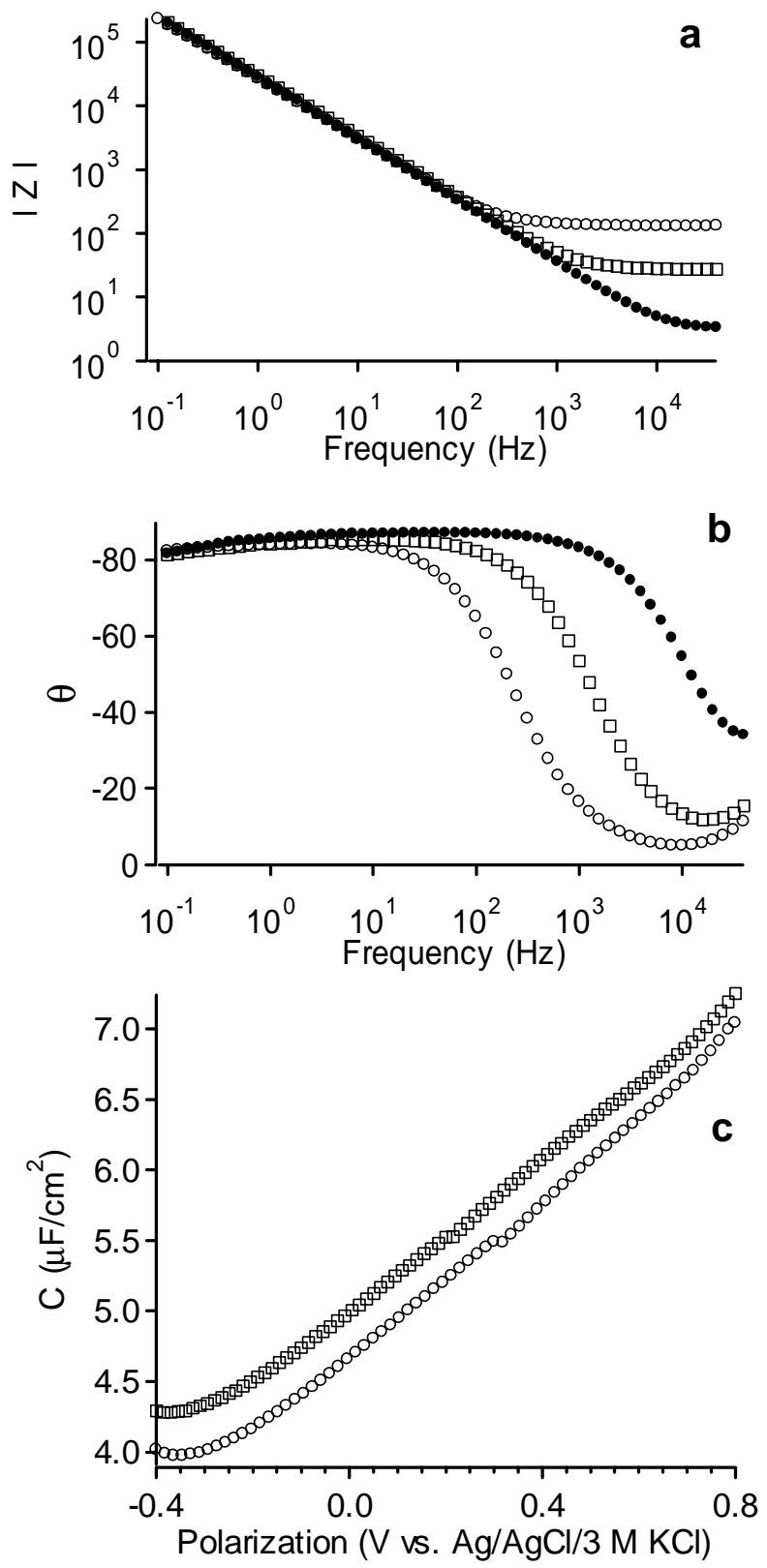

Figure 5: AC-impedance characterization of hydrogen terminated amorphous carbon surface. Impedance magnitude (a) and phase angle $\theta$ (b) as a function of frequency obtained in $0.01(\circ), 0.1(\square)$ and $1 \mathrm{M}(\bullet) \mathrm{KCl}$ solutions. (c) Capacitance vs. polarization plots collected at $10 \mathrm{~Hz}$ in $0.01(\circ)$ and $0.1 \mathrm{M}(\square) \mathrm{KCl}$ solutions. 


\section{HOMO/SOMO surfaces and spin densities of alkenes and their anions}

Figure 6 shows to the left the surfaces of the highest-occupied molecular orbital of the four alkenes as calculated at the B3LYP/D95 theory level ${ }^{15}$ (visualization achieved using Gabedit software). ${ }^{1}$ The HOMOs are mostly located on the olefin terminal group of the molecules except for $\mathrm{tBOC}$, where the $\mathrm{HOMO}$ has contributions also from the carbamate group. The HOMO surface suggests that a first ionization of the molecules is most likely to create a hole localized on the $\mathrm{C}=\mathrm{C} \pi$-states (except for $\mathrm{tBOC}$ ), common to all four molecules; hence, the small spread in the ionization potentials observed in our calculations (see text). The right hand side of Figure 6 shows the surfaces of the singlyoccupied molecular orbitals (SOMO) of the relaxed anions (adiabatic calculations). The atoms at the $\alpha$-terminus give the largest contributions to the SOMO, except for dodecene where the orbital is localized on the olefin group.

The unpaired spin density distributions are also a useful means of understanding the importance of the terminal groups for the stability of the radical anions. Figure 7 shows the spin distributions obtained from the vertical (left) and the adiabatic (right) electron affinity calculations. In both cases, except for dodecene, the spin density is mostly localized on the group at the $\alpha$-terminus, progressively shifts shifting toward the olefin terminus as the calculated EAs increase along the alkene series. The unpaired electron can also be found close to the olefin terminus in the vertical EA calculations, however, as the anion relaxes the spin density increasingly localizes on the $\alpha$-terminus. 
HOMO
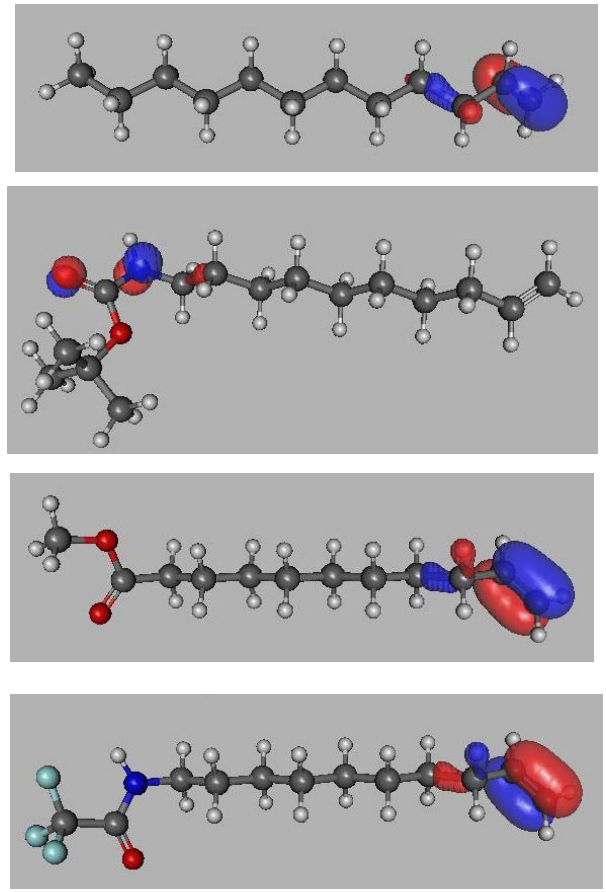

SOMO

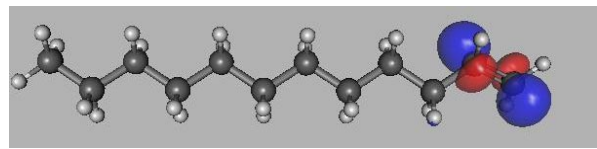

tBOC

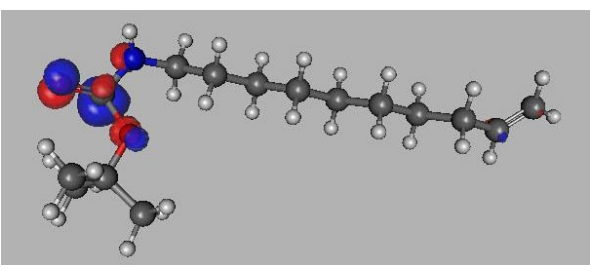

UA-Me

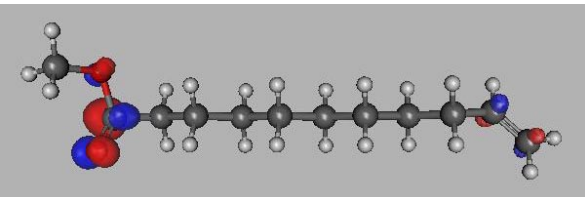

TFAAD

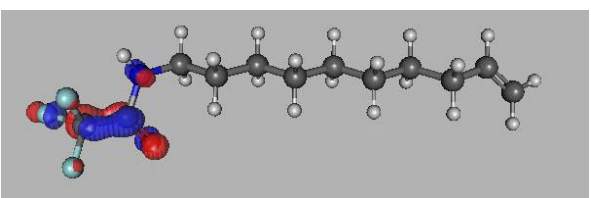

Figure 6: HOMO and SOMO surfaces of the optimized neutral molecule and radical anions calculated for the four alkenes (visualization was achieved using the Gabedit software). ${ }^{1}$ The olefin terminus is always displayed on the right hand side. 


\section{Optimized neutral geometry}
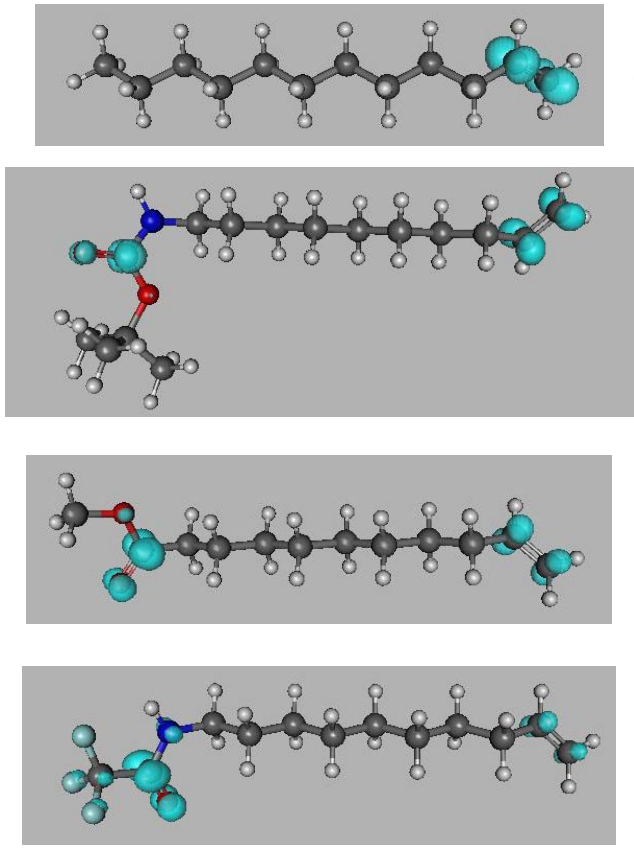

\section{Optimized anion geometry}

dodecene

tBOC
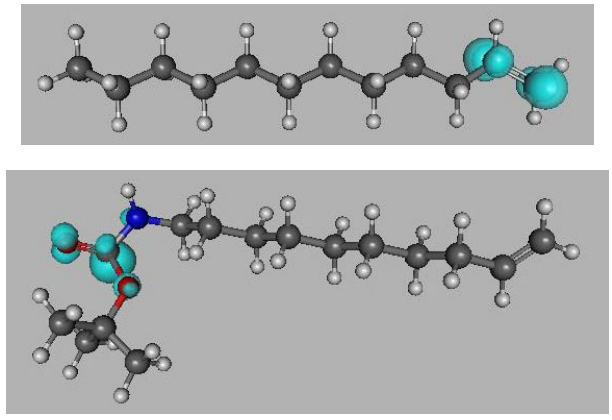

UA-Me

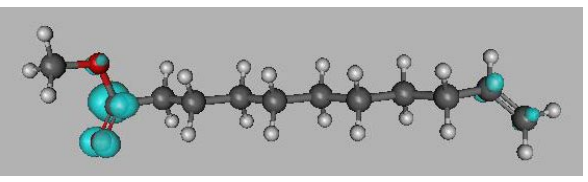

TFAAD

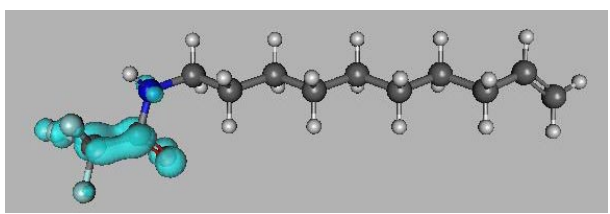

Figure 7: Unpaired spin density distributions obtained from the calculated radical anion structures at optimized neutral geometry (left) and optimized anion geometry (right) for the four alkenes (visualization was achieved using the Gabedit software). ${ }^{1}$ The olefin terminus is always displayed on the right hand side. 
Geometries and electronic energies of optimized neutral and ionic species

\begin{tabular}{|c|c|c|c|c|c|c|}
\hline & & eutral & -47 & $652194951 \mathrm{H}$ & & \\
\hline $\mathrm{H}$ & 1 & 2.488990 & & & & \\
\hline $\mathrm{H}$ & 2 & 2.600582 & 1 & 153.293591 & & \\
\hline $\mathrm{H}$ & 3 & 2.582821 & 2 & 179.506214 & 1 & -10.618739 \\
\hline X & 4 & 2.769777 & 3 & 158.922129 & 2 & -168.141169 \\
\hline $\mathrm{H}$ & 5 & 1.000000 & 4 & 68.785997 & 3 & 179.815637 \\
\hline $\mathrm{H}$ & 6 & 2.579449 & 5 & 89.999998 & 4 & -179.953953 \\
\hline $\mathrm{C}$ & 7 & 12.672120 & 6 & 6.774273 & 5 & 131.557184 \\
\hline $\mathrm{C}$ & 8 & 2.547664 & 7 & 23.179331 & 6 & -58.826524 \\
\hline $\mathrm{H}$ & 9 & 3.536186 & 8 & 8.683179 & 7 & -110.965000 \\
\hline $\mathrm{C}$ & 10 & 5.945684 & 9 & 11.509193 & 8 & \\
\hline $\mathrm{H}$ & 11 & 2.773933 & 10 & 38.312329 & 9 & 16.123222 \\
\hline $\mathrm{H}$ & 12 & 3.073666 & 11 & & 10 & \\
\hline $\mathrm{C}$ & 13 & 4.252654 & 12 & 89.863107 & 11 & 4.245603 \\
\hline $\mathrm{C}$ & 14 & 6.473141 & 13 & 18.724131 & 12 & 16336 \\
\hline $\mathrm{H}$ & 15 & 4.196599 & 14 & 18.08 & 13 & -178.364613 \\
\hline $\mathrm{H}$ & 16 & & 15 & & 14 & \\
\hline $\mathrm{C}$ & 17 & 4.246579 & 16 & 90.65 & 15 & -179.252563 \\
\hline $\mathrm{C}$ & 18 & 6.504063 & 17 & & 16 & \\
\hline $\mathrm{H}$ & 19 & 4.244512 & 18 & 37 & 17 & 663660 \\
\hline $\mathrm{H}$ & 20 & 3.079955 & 19 & 7703 & 18 & -4.025688 \\
\hline $\mathrm{C}$ & 21 & 4.247040 & 20 & 3602 & 19 & -179.848912 \\
\hline $\mathrm{C}$ & 22 & 6.506296 & 21 & & 20 & 4.230610 \\
\hline $\mathrm{H}$ & 23 & & 22 & & 21 & 9.847138 \\
\hline $\mathrm{H}$ & 24 & 3.080626 & 23 & 90.720091 & 22 & -4.167204 \\
\hline $\mathrm{C}$ & 25 & 2.806663 & 24 & & 23 & -4.365176 \\
\hline $\mathrm{H}$ & 26 & 6.087939 & 25 & & 24 & -8.432715 \\
\hline $\mathrm{H}$ & 27 & 2.520475 & 26 & 34.182387 & 25 & 174.787500 \\
\hline $\mathrm{H}$ & 28 & 3.093022 & 27 & 35.031098 & 26 & 1.516115 \\
\hline $\mathrm{C}$ & 29 & 2.837225 & 28 & 42.690778 & 27 & 176.786125 \\
\hline $\mathrm{C}$ & 30 & 2.574795 & 29 & 22.770504 & 28 & -19.462967 \\
\hline $\mathrm{H}$ & 31 & 12.988279 & 30 & 5.175329 & 29 & -108.056422 \\
\hline $\mathrm{H}$ & 32 & 2.626033 & 31 & 4.305337 & 30 & 170.136781 \\
\hline $\mathrm{H}$ & 33 & 2.579119 & 32 & 179.086519 & 31 & 122.258053 \\
\hline $\mathrm{H}$ & 34 & 2.578650 & 33 & 179.778689 & 32 & -2.256476 \\
\hline $\mathrm{H}$ & 35 & 2.581145 & 34 & 179.892184 & 33 & 9.237007 \\
\hline $\mathrm{H}$ & 36 & 2.615135 & 35 & 179.794355 & 34 & -45.600833 \\
\hline
\end{tabular}

Dodecene cation $\quad E=-471.340018985 \mathrm{Ha}$

$\begin{array}{lllllll}\mathrm{H} & & & & & & \\ \mathrm{H} & 1 & 13.382962 & & & & \\ \mathrm{H} & 2 & 10.799285 & 1 & 1.569790 & & \\ \mathrm{H} & 3 & 2.557294 & 2 & 6.941170 & 1 & 177.945235 \\ \mathrm{H} & 4 & 2.546258 & 3 & 179.575807 & 2 & -60.463548\end{array}$




$\begin{array}{lllllll}\mathrm{H} & 5 & 2.558897 & 4 & 178.914803 & 3 & -91.768953 \\ \mathrm{H} & 6 & 4.146399 & 5 & 176.150398 & 4 & -91.764342 \\ \mathrm{H} & 7 & 13.270073 & 6 & 7.032887 & 5 & -156.609358 \\ \mathrm{C} & 8 & 12.295746 & 7 & 2.167196 & 6 & -179.276402 \\ \mathrm{H} & 9 & 9.782081 & 8 & 2.160320 & 7 & 166.689023 \\ \mathrm{C} & 10 & 4.216708 & 9 & 146.677964 & 8 & -30.432547 \\ \mathrm{C} & 11 & 2.552253 & 10 & 25.055642 & 9 & -9.664070 \\ \mathrm{H} & 12 & 3.504293 & 11 & 10.573941 & 10 & -28.191945 \\ \mathrm{H} & 13 & 7.360498 & 12 & 19.222354 & 11 & 161.794422 \\ \mathrm{C} & 14 & 2.192498 & 13 & 45.357241 & 12 & 5.330757 \\ \mathrm{C} & 15 & 3.930602 & 14 & 113.286759 & 13 & 2.945036 \\ \mathrm{H} & 16 & 7.709266 & 15 & 19.502585 & 14 & 6.754968 \\ \mathrm{C} & 17 & 2.190172 & 16 & 47.447847 & 15 & 5.649470 \\ \mathrm{C} & 18 & 3.922064 & 17 & 113.240842 & 16 & 2.370675 \\ \mathrm{C} & 19 & 6.410546 & 18 & 5.293902 & 17 & 24.263647 \\ \mathrm{H} & 20 & 2.214037 & 19 & 118.226353 & 18 & -156.197835 \\ \mathrm{H} & 21 & 11.839604 & 20 & 41.175371 & 19 & -1.131061 \\ \mathrm{C} & 22 & 6.642836 & 21 & 1.961196 & 20 & -157.462372 \\ \mathrm{H} & 23 & 4.208701 & 22 & 9.509632 & 21 & 30.824777 \\ \mathrm{C} & 24 & 10.178655 & 23 & 19.137757 & 22 & -175.263300 \\ \mathrm{H} & 25 & 7.655389 & 24 & 2.033342 & 23 & -165.968065 \\ \mathrm{C} & 26 & 4.211782 & 25 & 17.457312 & 24 & 19.907311 \\ \mathrm{H} & 27 & 2.192403 & 26 & 29.565283 & 25 & 6.612693 \\ \mathrm{H} & 28 & 2.550567 & 27 & 54.492420 & 26 & 178.414044 \\ \mathrm{C} & 29 & 2.218791 & 28 & 125.547652 & 27 & -1.942519 \\ \mathrm{H} & 30 & 10.239312 & 29 & 56.138924 & 28 & -6.203434 \\ \mathrm{H} & 31 & 11.805415 & 30 & 8.119138 & 29 & -15.238633 \\ \mathrm{H} & 32 & 9.304334 & 31 & 2.623650 & 30 & -36.135892 \\ \mathrm{H} & 33 & 2.539179 & 32 & 11.770002 & 31 & -175.939292 \\ \mathrm{H} & 34 & 2.533792 & 33 & 179.425534 & 32 & -146.367725 \\ \mathrm{H} & 35 & 2.511589 & 34 & 179.408646 & 33 & -60.286767\end{array}$

\section{Dodecene anion $\quad E=-471.567987654 \mathrm{Ha}$}

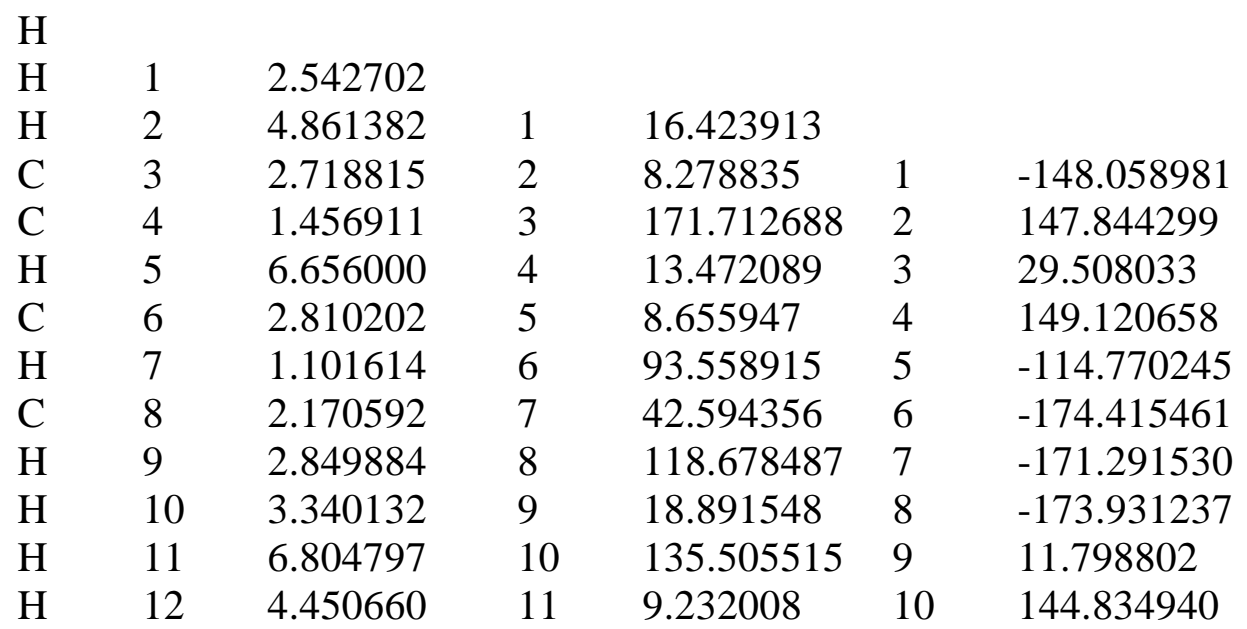




$\begin{array}{lllllll}\mathrm{C} & 13 & 2.174663 & 12 & 30.776830 & 11 & -37.621868 \\ \mathrm{C} & 14 & 1.545144 & 13 & 28.611356 & 12 & -173.779836 \\ \mathrm{H} & 15 & 2.171537 & 14 & 28.699342 & 13 & 179.579641 \\ \mathrm{H} & 16 & 4.403755 & 15 & 34.968547 & 14 & 172.608088 \\ \mathrm{H} & 17 & 9.532857 & 16 & 16.655292 & 15 & 17.973273 \\ \mathrm{H} & 18 & 4.450492 & 17 & 20.973887 & 16 & -177.076796 \\ \mathrm{C} & 19 & 2.174440 & 18 & 30.702247 & 17 & -15.869509 \\ \mathrm{C} & 20 & 1.544745 & 19 & 28.596715 & 18 & -173.193460 \\ \mathrm{H} & 21 & 2.171822 & 20 & 28.662527 & 19 & -179.881321 \\ \mathrm{H} & 22 & 4.445359 & 21 & 31.168548 & 20 & 173.438516 \\ \mathrm{H} & 23 & 5.476968 & 22 & 34.209714 & 21 & 17.137606 \\ \mathrm{H} & 24 & 4.473391 & 23 & 145.536488 & 22 & 1.030999 \\ \mathrm{C} & 25 & 2.839368 & 24 & 23.199144 & 23 & 16.091147 \\ \mathrm{C} & 26 & 1.544358 & 25 & 154.049516 & 24 & 7.715260 \\ \mathrm{H} & 27 & 2.172966 & 26 & 28.619119 & 25 & 166.656195 \\ \mathrm{H} & 28 & 4.438312 & 27 & 31.061290 & 26 & 173.477155 \\ \mathrm{H} & 29 & 5.462636 & 28 & 34.289770 & 27 & 17.233759 \\ \mathrm{C} & 30 & 2.174925 & 29 & 115.166101 & 28 & 9.942206 \\ \mathrm{C} & 31 & 1.543674 & 30 & 28.515561 & 29 & 17.375854 \\ \mathrm{H} & 32 & 2.809607 & 31 & 153.386676 & 30 & -167.382409 \\ \mathrm{H} & 33 & 4.462578 & 32 & 23.433544 & 31 & -8.305624 \\ \mathrm{H} & 34 & 1.776679 & 33 & 94.542912 & 32 & -22.768367 \\ \mathrm{H} & 35 & 2.522346 & 34 & 69.850313 & 33 & -15.164549\end{array}$

\begin{tabular}{lllllll} 
tBOC neutral & \multicolumn{6}{c}{$\mathbf{E}=\mathbf{- 7 9 4 . 1 6 6 1 6 0 2 2 4} \mathbf{H a}$} \\
$\mathrm{H}$ & \multicolumn{7}{c}{} & & \\
$\mathrm{H}$ & 1 & 2.569039 & & & \\
$\mathrm{H}$ & 2 & 2.575092 & 1 & 179.691025 & & \\
$\mathrm{H}$ & 3 & 6.295732 & 2 & 165.473945 & 1 & 171.676597 \\
$\mathrm{H}$ & 4 & 3.851825 & 3 & 9.826570 & 2 & 179.775896 \\
$\mathrm{H}$ & 5 & 9.537953 & 4 & 146.708776 & 3 & -10.425774 \\
$\mathrm{H}$ & 6 & 13.908801 & 5 & 3.605157 & 4 & -34.063990 \\
$\mathrm{C}$ & 7 & 1.087970 & 6 & 29.252631 & 5 & 157.806657 \\
$\mathrm{H}$ & 8 & 10.118662 & 7 & 152.309224 & 6 & 1.989538 \\
$\mathrm{C}$ & 9 & 2.173712 & 8 & 117.995509 & 7 & 12.756212 \\
$\mathrm{H}$ & 10 & 4.237319 & 9 & 29.711767 & 8 & 3.962343 \\
$\mathrm{C}$ & 11 & 2.172561 & 10 & 28.999523 & 9 & 178.498879 \\
$\mathrm{H}$ & 12 & 4.246620 & 11 & 29.332270 & 10 & 179.230574 \\
$\mathrm{C}$ & 13 & 2.172951 & 12 & 29.212959 & 11 & 178.779957 \\
$\mathrm{~N}$ & 14 & 6.434061 & 13 & 120.567934 & 12 & -4.080128 \\
$\mathrm{H}$ & 15 & 10.346868 & 14 & 11.884442 & 13 & -2.892610 \\
$\mathrm{C}$ & 16 & 2.181997 & 15 & 46.906298 & 14 & -4.878222 \\
$\mathrm{C}$ & 17 & 6.498579 & 16 & 120.635215 & 15 & -1.673475 \\
$\mathrm{H}$ & 18 & 2.171503 & 17 & 119.543988 & 16 & 177.644291 \\
$\mathrm{C}$ & 19 & 4.238304 & 18 & 29.349796 & 17 & 4.075078 \\
$\mathrm{C}$ & 20 & 6.471950 & 19 & 148.658347 & 18 & -174.563887
\end{tabular}




$\begin{array}{lllllll}\mathrm{H} & 21 & 7.774805 & 20 & 14.073774 & 19 & -3.336355 \\ \mathrm{C} & 22 & 4.243807 & 21 & 17.236221 & 20 & -5.532256 \\ \mathrm{H} & 23 & 2.172561 & 22 & 29.240212 & 21 & -9.290643 \\ \mathrm{C} & 24 & 4.247000 & 23 & 29.218520 & 22 & 179.596782 \\ \mathrm{H} & 25 & 2.177756 & 24 & 29.289663 & 23 & 178.808672 \\ \mathrm{H} & 26 & 2.626326 & 25 & 54.012878 & 24 & -179.038243 \\ \mathrm{C} & 27 & 12.393760 & 26 & 9.316649 & 25 & 43.801373 \\ \mathrm{H} & 28 & 3.175564 & 27 & 13.283993 & 26 & -155.793743 \\ \mathrm{O} & 29 & 4.230726 & 28 & 10.606230 & 27 & -176.968131 \\ \mathrm{H} & 30 & 6.811642 & 29 & 2.580224 & 28 & 159.324262 \\ \mathrm{H} & 31 & 2.580330 & 30 & 175.958393 & 29 & -177.739462 \\ \mathrm{H} & 32 & 2.544567 & 31 & 179.453428 & 30 & -39.015130 \\ \mathrm{O} & 33 & 10.360020 & 32 & 7.948400 & 31 & 138.037475 \\ \mathrm{H} & 34 & 2.698160 & 33 & 143.411542 & 32 & 154.251344 \\ \mathrm{H} & 35 & 1.782401 & 34 & 74.190083 & 33 & 160.095182 \\ \mathrm{C} & 36 & 1.092804 & 35 & 35.597785 & 34 & -73.159230 \\ \mathrm{C} & 37 & 1.540678 & 36 & 110.945031 & 35 & 121.740376 \\ \mathrm{H} & 38 & 2.178939 & 37 & 94.762296 & 36 & -155.096704 \\ \mathrm{H} & 39 & 4.335451 & 38 & 6.474471 & 37 & -141.710318 \\ \mathrm{C} & 40 & 3.495388 & 39 & 10.373870 & 38 & 164.711352 \\ \mathrm{H} & 41 & 1.095994 & 40 & 95.959180 & 39 & 123.326940 \\ \mathrm{H} & 42 & 3.802913 & 41 & 19.226243 & 40 & 20.494071 \\ \mathrm{C} & 43 & 2.838310 & 42 & 46.479774 & 41 & -164.884625 \\ \mathrm{H} & 44 & 1.097060 & 43 & 152.119212 & 42 & -8.165582 \\ \mathrm{H} & 45 & 3.156453 & 44 & 62.017338 & 43 & -12.384943 \\ \mathrm{H} & 46 & 2.613134 & 45 & 34.282673 & 44 & -41.595841\end{array}$

\begin{tabular}{lllllll} 
tBOC cation & \multicolumn{5}{c}{$\mathbf{E}=\mathbf{- 7 9 3 . 8 7 1 2 5 2 7 5 3 ~ H a}$} \\
$\mathrm{H}$ & & & & & \\
$\mathrm{H}$ & 1 & 1.785814 & & & & \\
$\mathrm{H}$ & 2 & 1.772786 & 1 & 59.762079 & & \\
$\mathrm{C}$ & 3 & 1.096812 & 2 & 35.897043 & 1 & -36.639425 \\
$\mathrm{H}$ & 4 & 12.060004 & 3 & 132.478899 & 2 & 144.924934 \\
$\mathrm{H}$ & 5 & 1.778997 & 4 & 66.323885 & 3 & 15.891627 \\
$\mathrm{O}$ & 6 & 11.929931 & 5 & 91.103116 & 4 & 4.677934 \\
$\mathrm{H}$ & 7 & 9.556216 & 6 & 10.606842 & 5 & 2.943019 \\
$\mathrm{H}$ & 8 & 1.767706 & 7 & 79.661041 & 6 & 3.170174 \\
$\mathrm{H}$ & 9 & 3.115007 & 8 & 55.406828 & 7 & 2.479461 \\
$\mathrm{H}$ & 10 & 1.768079 & 9 & 55.378395 & 8 & -179.999946 \\
$\mathrm{H}$ & 11 & 3.127682 & 10 & 55.056942 & 9 & -179.810387 \\
$\mathrm{H}$ & 12 & 1.776267 & 11 & 55.565814 & 10 & -179.412217 \\
$\mathrm{C}$ & 13 & 7.792105 & 12 & 83.038845 & 11 & -4.559115 \\
$\mathrm{C}$ & 14 & 10.974213 & 13 & 2.910265 & 12 & -69.459009 \\
$\mathrm{C}$ & 15 & 2.540379 & 14 & 97.624078 & 13 & -69.493103 \\
$\mathrm{C}$ & 16 & 9.174909 & 15 & 64.888798 & 14 & -0.751598 \\
$\mathrm{H}$ & 17 & 7.839579 & 16 & 11.658412 & 15 & -177.444092
\end{tabular}




$\begin{array}{lllllll}\mathrm{H} & 18 & 5.626479 & 17 & 71.160087 & 16 & 3.678311 \\ \mathrm{C} & 19 & 5.503404 & 18 & 64.161926 & 17 & 0.852591 \\ \mathrm{C} & 20 & 2.548114 & 19 & 10.076148 & 18 & 13.117300 \\ \mathrm{O} & 21 & 3.475930 & 20 & 139.986035 & 19 & -167.219697 \\ \mathrm{H} & 22 & 2.815637 & 21 & 154.467348 & 20 & 176.090512 \\ \mathrm{H} & 23 & 15.851977 & 22 & 3.513440 & 21 & 4.332333 \\ \mathrm{~N} & 24 & 12.329154 & 23 & 9.063337 & 22 & 178.672489 \\ \mathrm{H} & 25 & 5.654060 & 24 & 100.543321 & 23 & -2.788443 \\ \mathrm{C} & 26 & 1.096187 & 25 & 18.961657 & 24 & -27.371779 \\ \mathrm{H} & 27 & 2.800387 & 26 & 69.933131 & 25 & -29.088880 \\ \mathrm{C} & 28 & 1.096791 & 27 & 65.535319 & 26 & 178.407577 \\ \mathrm{C} & 29 & 14.095472 & 28 & 127.692059 & 27 & -1.650998 \\ \mathrm{C} & 30 & 1.374874 & 29 & 146.077241 & 28 & -178.367706 \\ \mathrm{C} & 31 & 3.569830 & 30 & 32.773839 & 29 & -1.610463 \\ \mathrm{C} & 32 & 2.556183 & 31 & 163.807166 & 30 & 177.175615 \\ \mathrm{C} & 33 & 2.564165 & 32 & 179.822701 & 31 & 139.687275 \\ \mathrm{C} & 34 & 2.563092 & 33 & 179.598237 & 32 & -62.776863 \\ \mathrm{H} & 35 & 10.433829 & 34 & 6.204307 & 33 & 66.273738 \\ \mathrm{H} & 36 & 2.463020 & 35 & 126.132637 & 34 & -34.818885 \\ \mathrm{H} & 37 & 4.302339 & 36 & 48.725294 & 35 & -2.952767 \\ \mathrm{H} & 38 & 1.769411 & 37 & 90.060777 & 36 & -5.064517 \\ \mathrm{H} & 39 & 3.125091 & 38 & 55.391573 & 37 & -179.226213 \\ \mathrm{H} & 40 & 1.767835 & 39 & 55.299874 & 38 & 179.561647 \\ \mathrm{H} & 41 & 7.038093 & 40 & 120.126380 & 39 & 177.480162 \\ \mathrm{H} & 42 & 6.366912 & 41 & 26.167403 & 40 & 2.335808 \\ \mathrm{H} & 43 & 1.769449 & 42 & 33.324718 & 41 & 2.740307 \\ \mathrm{H} & 44 & 2.573848 & 43 & 91.117569 & 42 & 2.507090 \\ \mathrm{H} & 45 & 4.175777 & 44 & 143.178427 & 43 & -178.706886 \\ \mathrm{H} & 46 & 5.483616 & 45 & 14.773138 & 44 & -178.759369\end{array}$

\begin{tabular}{lllllll} 
tBOC anion & \multicolumn{5}{c}{$\mathbf{E}=\mathbf{- 7 9 4 . 1 0 7 6 3 9 6 4 2 ~ H a}$} \\
$\mathrm{H}$ & & & & & \\
$\mathrm{H}$ & 1 & 12.629016 & & & \\
$\mathrm{H}$ & 2 & 2.640265 & 1 & 14.670130 & & \\
$\mathrm{H}$ & 3 & 2.590470 & 2 & 179.722239 & 1 & 13.445662 \\
$\mathrm{H}$ & 4 & 2.594214 & 3 & 179.158437 & 2 & 170.139971 \\
$\mathrm{H}$ & 5 & 4.332597 & 4 & 118.160871 & 3 & -169.323461 \\
$\mathrm{H}$ & 6 & 3.118297 & 5 & 133.110653 & 4 & -178.481201 \\
$\mathrm{H}$ & 7 & 5.306205 & 6 & 47.417939 & 5 & -1.075078 \\
$\mathrm{C}$ & 8 & 4.256610 & 7 & 4.046550 & 6 & -146.915744 \\
$\mathrm{H}$ & 9 & 15.322053 & 8 & 39.199264 & 7 & 37.110070 \\
$\mathrm{H}$ & 10 & 4.450992 & 9 & 20.801182 & 8 & 6.809726 \\
$\mathrm{H}$ & 11 & 2.596397 & 10 & 177.809575 & 9 & 36.917877 \\
$\mathrm{H}$ & 12 & 9.486368 & 11 & 151.388416 & 10 & -37.080762 \\
$\mathrm{C}$ & 13 & 13.920462 & 12 & 14.315299 & 11 & 7.225954 \\
$\mathrm{H}$ & 14 & 7.818875 & 13 & 22.589607 & 12 & 3.775747
\end{tabular}




\begin{tabular}{|c|c|c|c|c|c|c|}
\hline $\mathrm{C}$ & 15 & 5.287822 & 14 & 3.500664 & 13 & 15.227792 \\
\hline $\mathrm{H}$ & 16 & 9.849204 & 15 & 48.067539 & 14 & -167.484433 \\
\hline $\mathrm{C}$ & 17 & 7.913747 & 16 & 11.127102 & 15 & -4.585658 \\
\hline $\mathrm{H}$ & 18 & 5.261435 & 17 & 60.284824 & 16 & 177.326626 \\
\hline $\mathrm{C}$ & 19 & 11.297736 & 18 & 10.972183 & 17 & 175.946030 \\
\hline $\mathrm{C}$ & 20 & 8.780349 & 19 & 7.011518 & 18 & -7.849124 \\
\hline $\mathrm{C}$ & 21 & 5.411735 & 20 & 123.206149 & 19 & 176.619711 \\
\hline $\mathrm{C}$ & 22 & 4.846055 & 21 & 28.455370 & 20 & 176.531617 \\
\hline $\mathrm{C}$ & 23 & 4.261495 & 22 & 18.115902 & 21 & -165.661919 \\
\hline $\mathrm{C}$ & 24 & 10.953286 & 23 & 53.384091 & 22 & 20.609993 \\
\hline $\mathrm{C}$ & 25 & 2.583068 & 24 & 19.808689 & 23 & 12.571713 \\
\hline $\mathrm{O}$ & 26 & 7.633884 & 25 & 162.282319 & 24 & 1.210753 \\
\hline $\mathrm{C}$ & 27 & 1.589615 & 26 & 116.974719 & 25 & -176.553675 \\
\hline $\mathrm{C}$ & 28 & 5.928178 & 27 & 56.842119 & 26 & -0.743238 \\
\hline $\mathrm{H}$ & 29 & 5.354074 & 28 & 43.016346 & 27 & -4.313888 \\
\hline $\mathrm{C}$ & 30 & 4.381726 & 29 & 28.581685 & 28 & -3.875967 \\
\hline $\mathrm{H}$ & 31 & 7.780561 & 30 & 89.406441 & 29 & -3.083027 \\
\hline $\mathrm{H}$ & 32 & 3.538576 & 31 & 122.455079 & 30 & 179.526915 \\
\hline $\mathrm{N}$ & 33 & 12.630686 & 32 & 42.641171 & 31 & 0.317881 \\
\hline $\mathrm{H}$ & 34 & 7.790769 & 33 & 15.504093 & 32 & 1.812210 \\
\hline $\mathrm{H}$ & 35 & 2.591840 & 34 & 8.830042 & 33 & 165.978796 \\
\hline $\mathrm{H}$ & 36 & 2.640573 & 35 & 179.026786 & 34 & 81.681867 \\
\hline $\mathrm{H}$ & 37 & 8.041835 & 36 & 12.415302 & 35 & -113.018041 \\
\hline $\mathrm{O}$ & 38 & 12.253754 & 37 & 3.678505 & 36 & -153.603080 \\
\hline $\mathrm{H}$ & 39 & 9.701560 & 38 & 2.512698 & 37 & -152.866769 \\
\hline $\mathrm{H}$ & 40 & 10.495944 & 39 & 22.211224 & 38 & -18.438515 \\
\hline $\mathrm{H}$ & 41 & 8.476388 & 40 & 9.687334 & 39 & -7.208350 \\
\hline $\mathrm{C}$ & 42 & 7.529687 & 41 & 4.055402 & 40 & -170.928565 \\
\hline $\mathrm{H}$ & 43 & 5.756530 & 42 & 16.165435 & 41 & -171.462704 \\
\hline $\mathrm{H}$ & 44 & 2.698912 & 43 & 55.112675 & 42 & 175.857083 \\
\hline $\mathrm{H}$ & 45 & 4.322438 & 44 & 109.172343 & 43 & 4.265192 \\
\hline $\mathrm{H}$ & 46 & 1.780065 & 45 & 93.051764 & 44 & 14.021596 \\
\hline
\end{tabular}

\begin{tabular}{|c|c|c|c|c|c|c|}
\hline \multicolumn{5}{|c|}{$\begin{array}{l}\text { UA-Me neutral } \\
\mathrm{H}\end{array}$} & & \\
\hline $\mathrm{H}$ & 1 & 2.488824 & & & & \\
\hline $\mathrm{H}$ & 2 & 2.600698 & 1 & 153.268504 & & \\
\hline $\mathrm{H}$ & 3 & 2.580795 & 2 & 179.378201 & 1 & -4.948749 \\
\hline $\mathrm{H}$ & 4 & 2.579453 & 3 & 179.563173 & 2 & 9.397776 \\
\hline $\mathrm{H}$ & 5 & 4.465428 & 4 & 149.974594 & 3 & 174.151403 \\
\hline $\mathrm{C}$ & 6 & 14.291886 & 5 & 17.067028 & 4 & 14.492765 \\
\hline $\mathrm{C}$ & 7 & 2.547521 & 6 & 15.041092 & 5 & 174.799551 \\
\hline $\mathrm{H}$ & 8 & 1.102884 & 7 & 118.949535 & 6 & 169.391217 \\
\hline $\mathrm{O}$ & 9 & 11.104969 & 8 & 82.514464 & 7 & -179.248213 \\
\hline $\mathrm{C}$ & 10 & 8.452996 & 9 & 4.795965 & 8 & -15.922941 \\
\hline $\mathrm{H}$ & 11 & 1.102169 & 10 & 94.862994 & 9 & -17.066447 \\
\hline
\end{tabular}




$\begin{array}{lllllll}\mathrm{H} & 12 & 6.303281 & 11 & 66.226508 & 10 & -178.122329 \\ \mathrm{C} & 13 & 8.456138 & 12 & 14.136857 & 11 & 9.075713 \\ \mathrm{H} & 14 & 1.101824 & 13 & 99.787962 & 12 & 8.539799 \\ \mathrm{C} & 15 & 6.637449 & 14 & 76.605977 & 13 & 1.247258 \\ \mathrm{C} & 16 & 9.029239 & 15 & 10.776850 & 14 & 9.396475 \\ \mathrm{H} & 17 & 6.679968 & 16 & 11.274872 & 15 & -178.983916 \\ \mathrm{H} & 18 & 7.035013 & 17 & 8.698011 & 16 & -8.276407 \\ \mathrm{C} & 19 & 6.681534 & 18 & 8.715817 & 17 & 179.775281 \\ \mathrm{C} & 20 & 10.764230 & 19 & 1.279164 & 18 & 22.783975 \\ \mathrm{C} & 21 & 2.439031 & 20 & 39.475881 & 19 & -164.288361 \\ \mathrm{H} & 22 & 6.623423 & 21 & 141.465105 & 20 & -0.542231 \\ \mathrm{C} & 23 & 1.101820 & 22 & 76.395421 & 21 & -6.941119 \\ \mathrm{H} & 24 & 8.993941 & 23 & 101.638764 & 22 & -1.167107 \\ \mathrm{H} & 25 & 6.894229 & 24 & 13.534157 & 23 & -7.997718 \\ \mathrm{C} & 26 & 1.101657 & 25 & 64.894006 & 24 & -8.547071 \\ \mathrm{H} & 27 & 6.360825 & 26 & 122.338421 & 25 & -0.045481 \\ \mathrm{O} & 28 & 2.047930 & 27 & 20.448871 & 26 & -10.329789 \\ \mathrm{H} & 29 & 3.291918 & 28 & 171.152738 & 27 & 170.109194 \\ \mathrm{C} & 30 & 1.095489 & 29 & 29.218106 & 28 & 174.809398 \\ \mathrm{H} & 31 & 10.411048 & 30 & 92.428965 & 29 & 172.049969 \\ \mathrm{H} & 32 & 2.626547 & 31 & 5.514000 & 30 & 99.889442 \\ \mathrm{H} & 33 & 2.579247 & 32 & 179.030225 & 31 & 122.676300 \\ \mathrm{H} & 34 & 2.579722 & 33 & 179.689418 & 32 & -16.601402 \\ \mathrm{H} & 35 & 2.578397 & 34 & 179.283510 & 33 & -7.905140\end{array}$

\begin{tabular}{lllllll}
\multicolumn{6}{l}{ UA-Me cation } & \multicolumn{6}{c}{$\mathbf{E}=\mathbf{- 6 2 0 . 5 6 7 6 3 3 8 0 2} \mathbf{H a}$} \\
$\mathrm{H}$ & & & & & \\
$\mathrm{H}$ & 1 & 2.532193 & & & & \\
$\mathrm{H}$ & 2 & 2.555085 & 1 & 136.079979 & & \\
$\mathrm{H}$ & 3 & 2.554473 & 2 & 179.288846 & 1 & 158.987642 \\
$\mathrm{H}$ & 4 & 2.553953 & 3 & 179.848989 & 2 & -91.704555 \\
$\mathrm{H}$ & 5 & 4.486929 & 4 & 154.246717 & 3 & 109.235959 \\
$\mathrm{C}$ & 6 & 14.198798 & 5 & 12.332823 & 4 & 17.306322 \\
$\mathrm{C}$ & 7 & 2.540837 & 6 & 24.139280 & 5 & 179.107777 \\
$\mathrm{H}$ & 8 & 3.525497 & 7 & 8.844684 & 6 & -127.803556 \\
$\mathrm{C}$ & 9 & 5.824772 & 8 & 14.635951 & 7 & 77.177625 \\
$\mathrm{H}$ & 10 & 2.757898 & 9 & 42.497780 & 8 & 15.104756 \\
$\mathrm{C}$ & 11 & 5.199583 & 10 & 11.503427 & 9 & 167.993129 \\
$\mathrm{H}$ & 12 & 4.231741 & 11 & 36.381371 & 10 & -16.963425 \\
$\mathrm{O}$ & 13 & 10.011188 & 12 & 18.194928 & 11 & -179.849271 \\
$\mathrm{H}$ & 14 & 8.839870 & 13 & 17.493900 & 12 & 3.111036 \\
$\mathrm{C}$ & 15 & 4.146758 & 14 & 144.510162 & 13 & 1.856599 \\
$\mathrm{C}$ & 16 & 8.925994 & 15 & 20.086580 & 14 & 1.702257 \\
$\mathrm{H}$ & 17 & 4.224120 & 16 & 20.438974 & 15 & -179.879455 \\
$\mathrm{H}$ & 18 & 3.087943 & 17 & 41.162387 & 16 & -4.442501 \\
$\mathrm{C}$ & 19 & 4.213121 & 18 & 41.164506 & 17 & 179.981542
\end{tabular}




$\begin{array}{lllllll}\mathrm{H} & 20 & 5.219433 & 19 & 36.268414 & 18 & 4.339515 \\ \mathrm{C} & 21 & 6.364421 & 20 & 131.367355 & 19 & 0.374475 \\ \mathrm{C} & 22 & 2.450984 & 21 & 22.451992 & 20 & 174.503384 \\ \mathrm{H} & 23 & 2.773434 & 22 & 97.586200 & 21 & -5.756736 \\ \mathrm{C} & 24 & 4.236766 & 23 & 132.426208 & 22 & 177.373297 \\ \mathrm{H} & 25 & 5.258487 & 24 & 36.026869 & 23 & 0.898602 \\ \mathrm{H} & 26 & 5.353679 & 25 & 103.103593 & 24 & 0.746347 \\ \mathrm{O} & 27 & 2.052753 & 26 & 4.965425 & 25 & 36.709703 \\ \mathrm{C} & 28 & 4.341490 & 27 & 146.118101 & 26 & -140.096466 \\ \mathrm{C} & 29 & 2.540532 & 28 & 28.116761 & 27 & -175.623634 \\ \mathrm{H} & 30 & 4.158361 & 29 & 143.623346 & 28 & 7.726101 \\ \mathrm{H} & 31 & 13.949558 & 30 & 27.268482 & 29 & 9.546080 \\ \mathrm{H} & 32 & 2.719930 & 31 & 14.175657 & 30 & 30.462198 \\ \mathrm{H} & 33 & 2.561433 & 32 & 175.942025 & 31 & 6.531141 \\ \mathrm{H} & 34 & 2.560490 & 33 & 179.793658 & 32 & 12.963497 \\ \mathrm{H} & 35 & 2.536800 & 34 & 179.082121 & 33 & 175.035492\end{array}$

\begin{tabular}{|c|c|c|c|c|c|c|}
\hline $\begin{array}{l}\mathbf{U} \\
\mathrm{H}\end{array}$ & e an & $\mathbf{E}=$ & 0 & 63139 На & & \\
\hline $\mathrm{H}$ & 1 & 2.482970 & & & & \\
\hline $\mathrm{H}$ & 2 & 2.616144 & 1 & 153.353704 & & \\
\hline $\mathrm{H}$ & 3 & 2.599029 & 2 & 179.421605 & 1 & -101.510484 \\
\hline $\mathrm{H}$ & 4 & 2.613270 & 3 & 179.337376 & 2 & 74.763308 \\
\hline $\mathrm{H}$ & 5 & 4.296517 & 4 & 148.914322 & 3 & -156.310615 \\
\hline $\mathrm{O}$ & 6 & 2.430278 & 5 & 38.686752 & 4 & -172.349652 \\
\hline $\mathrm{C}$ & 7 & 13.008577 & 6 & 114.108879 & 5 & -2.505818 \\
\hline $\mathrm{C}$ & 8 & 2.561174 & 7 & 23.409793 & 6 & -7.620609 \\
\hline $\mathrm{H}$ & 9 & 1.105643 & 8 & 120.145626 & 7 & 171.577287 \\
\hline $\mathrm{C}$ & 10 & 2.763596 & 9 & 69.347372 & 8 & 177.657206 \\
\hline $\mathrm{H}$ & 11 & 1.102925 & 10 & 66.126851 & 9 & -177.685764 \\
\hline $\mathrm{H}$ & 12 & 6.327670 & 11 & 66.421184 & 10 & 7.821880 \\
\hline $\mathrm{C}$ & 13 & 8.495836 & 12 & 13.931212 & 11 & 9.129457 \\
\hline $\mathrm{H}$ & 14 & 1.102411 & 13 & 99.375705 & 12 & 9.219541 \\
\hline $\mathrm{C}$ & 15 & 6.651380 & 14 & 77.103325 & 13 & 1.381873 \\
\hline $\mathrm{C}$ & 16 & 9.066493 & 15 & 10.615784 & 14 & 10.304563 \\
\hline $\mathrm{H}$ & 17 & 6.718405 & 16 & 11.406121 & 15 & -177.644481 \\
\hline $\mathrm{C}$ & 18 & 1.100508 & 17 & 75.005636 & 16 & -7.283836 \\
\hline $\mathrm{H}$ & 19 & 6.708863 & 18 & 105.013559 & 17 & -0.115553 \\
\hline $\mathrm{C}$ & 20 & 3.980732 & 19 & 178.912813 & 18 & -48.232177 \\
\hline $\mathrm{H}$ & 21 & 8.616856 & 20 & 11.959086 & 19 & 38.532037 \\
\hline $\mathrm{C}$ & 22 & 6.671103 & 21 & 11.473785 & 20 & 176.510896 \\
\hline $\mathrm{C}$ & 23 & 6.506357 & 22 & 9.491285 & 21 & 6.862392 \\
\hline $\mathrm{H}$ & 24 & 8.538967 & 23 & 20.867126 & 22 & 179.184952 \\
\hline $\mathrm{H}$ & 25 & 1.798072 & 24 & 100.561709 & 23 & -4.143703 \\
\hline $\mathrm{H}$ & 26 & 6.998749 & 25 & 82.080598 & 24 & -1.129396 \\
\hline $\mathrm{C}$ & 27 & 1.103611 & 26 & 62.759753 & 25 & -4.219472 \\
\hline
\end{tabular}




$\begin{array}{lllllll}\mathrm{O} & 28 & 4.538345 & 27 & 113.997229 & 26 & -3.602406 \\ \mathrm{H} & 29 & 3.354248 & 28 & 37.933414 & 27 & -5.489718 \\ \mathrm{C} & 30 & 1.101304 & 29 & 27.205153 & 28 & -9.659067 \\ \mathrm{H} & 31 & 10.471202 & 30 & 91.355001 & 29 & 176.067505 \\ \mathrm{H} & 32 & 2.651635 & 31 & 5.198779 & 30 & 92.637528 \\ \mathrm{H} & 33 & 2.577991 & 32 & 178.579920 & 31 & 111.765477 \\ \mathrm{H} & 34 & 2.582388 & 33 & 179.139244 & 32 & 4.491747 \\ \mathrm{H} & 35 & 2.614035 & 34 & 179.075865 & 33 & -18.143676\end{array}$

\begin{tabular}{|c|c|c|c|c|c|c|}
\hline $\begin{array}{l}\text { TF } \\
\mathrm{H}\end{array}$ & D & $\mathbf{E}=$ & 0 & 90269 На & & \\
\hline $\mathrm{H}$ & 1 & 3.841832 & & & & \\
\hline $\mathrm{H}$ & 2 & 6.281527 & 1 & 9.944490 & & \\
\hline $\mathrm{H}$ & 3 & 7.008013 & 2 & 14.762189 & 1 & -4.482205 \\
\hline $\mathrm{O}$ & 4 & 13.781911 & 3 & 8.779671 & 2 & -1.552109 \\
\hline $\mathrm{H}$ & 5 & 4.550900 & 4 & 18.714610 & 3 & -2.021369 \\
\hline $\mathrm{H}$ & 6 & 2.571994 & 5 & 27.327534 & 4 & -178.353259 \\
\hline $\mathrm{C}$ & 7 & 11.291753 & 6 & 3.667049 & 5 & 37.904747 \\
\hline $\mathrm{F}$ & 8 & 15.581624 & 7 & 8.603326 & 6 & -170.601284 \\
\hline $\mathrm{C}$ & 9 & 12.130449 & 8 & 4.916437 & 7 & -59.471026 \\
\hline $\mathrm{C}$ & 10 & 2.552640 & 9 & 170.849186 & 8 & -34.828024 \\
\hline $\mathrm{C}$ & 11 & 5.128915 & 10 & 0.706509 & 9 & -100.336566 \\
\hline $\mathrm{C}$ & 12 & 7.167292 & 11 & 166.997838 & 10 & 76.473407 \\
\hline $\mathrm{C}$ & 13 & 4.696466 & 12 & 7.217339 & 11 & 177.264934 \\
\hline $\mathrm{C}$ & 14 & 2.557461 & 13 & 20.711122 & 12 & 179.210170 \\
\hline $\mathrm{H}$ & 15 & 9.189376 & 14 & 10.714997 & 13 & 8.437359 \\
\hline $\mathrm{H}$ & 16 & 2.596251 & 15 & 11.189924 & 14 & 172.885362 \\
\hline $\mathrm{H}$ & 17 & 4.823146 & 16 & 35.620878 & 15 & -15.990293 \\
\hline $\mathrm{H}$ & 18 & 2.630381 & 17 & 35.358893 & 16 & 176.285642 \\
\hline $\mathrm{H}$ & 19 & 4.774593 & 18 & 144.391062 & 17 & 1.608779 \\
\hline $\mathrm{H}$ & 20 & 3.079938 & 19 & 29.380500 & 18 & 179.023523 \\
\hline $\mathrm{H}$ & 21 & 2.574141 & 20 & 65.267087 & 19 & -179.987340 \\
\hline $\mathrm{H}$ & 22 & 3.084229 & 21 & 114.937993 & 20 & 0.866977 \\
\hline $\mathrm{H}$ & 23 & 3.078940 & 22 & 49.464903 & 21 & -179.941784 \\
\hline $\mathrm{C}$ & 24 & 9.190726 & 23 & 54.501909 & 22 & -1.325252 \\
\hline $\mathrm{C}$ & 25 & 2.578367 & 24 & 10.910370 & 23 & -10.173975 \\
\hline $\mathrm{X}$ & 26 & 2.764629 & 25 & 158.755881 & 24 & 120.653716 \\
\hline $\mathrm{C}$ & 27 & 1.000000 & 26 & 68.818500 & 25 & 179.507583 \\
\hline $\mathrm{C}$ & 28 & 2.572403 & 27 & 89.999998 & 26 & -179.931979 \\
\hline $\mathrm{N}$ & 29 & 2.517871 & 28 & 178.117694 & 27 & -88.873230 \\
\hline $\mathrm{C}$ & 30 & 2.446927 & 29 & 138.836691 & 28 & 142.352486 \\
\hline $\mathrm{F}$ & 31 & 1.391262 & 30 & 124.506727 & 29 & 12.392717 \\
\hline $\mathrm{H}$ & 32 & 3.562846 & 31 & 34.744734 & 30 & 28.694313 \\
\hline $\mathrm{H}$ & 33 & 10.617053 & 32 & 110.746268 & 31 & -140.567516 \\
\hline $\mathrm{H}$ & 34 & 2.546869 & 33 & 1.962480 & 32 & -56.030002 \\
\hline$X$ & 35 & 2.765440 & 34 & 158.504631 & 33 & 82.859194 \\
\hline
\end{tabular}




$\begin{array}{lllllll}\mathrm{H} & 36 & 1.000000 & 35 & 68.843353 & 34 & 178.754025 \\ \mathrm{H} & 37 & 2.579374 & 36 & 89.999998 & 35 & -179.959317 \\ \mathrm{~F} & 38 & 4.744796 & 37 & 169.000592 & 36 & 56.953589\end{array}$

\begin{tabular}{lllllll} 
TFAAD cation & \multicolumn{1}{c}{$\mathbf{E}=\mathbf{- 8 9 8 . 4 3 2 2 7 8 4 2 1} \mathbf{H a}$} & & \\
$\mathrm{H}$ & & & & & & \\
$\mathrm{F}$ & 1 & 12.318543 & & & & \\
$\mathrm{H}$ & 2 & 9.827890 & 1 & 1.640038 & & \\
$\mathrm{~F}$ & 3 & 9.683311 & 2 & 13.243905 & 1 & 11.227894 \\
$\mathrm{H}$ & 4 & 7.350792 & 3 & 7.123593 & 2 & 3.294620 \\
$\mathrm{H}$ & 5 & 2.598315 & 4 & 26.316854 & 3 & 178.612874 \\
$\mathrm{H}$ & 6 & 2.996882 & 5 & 170.738880 & 4 & -105.543018 \\
$\mathrm{H}$ & 7 & 10.756035 & 6 & 13.808385 & 5 & 46.962864 \\
$\mathrm{C}$ & 8 & 11.853274 & 7 & 11.806568 & 6 & 128.105216 \\
$\mathrm{H}$ & 9 & 9.311706 & 8 & 1.290992 & 7 & 20.953738 \\
$\mathrm{C}$ & 10 & 2.789428 & 9 & 162.755135 & 8 & -165.384746 \\
$\mathrm{H}$ & 11 & 5.230550 & 10 & 10.723369 & 9 & -3.728962 \\
$\mathrm{C}$ & 12 & 2.775543 & 11 & 11.534981 & 10 & -178.449414 \\
$\mathrm{H}$ & 13 & 5.202234 & 12 & 10.421970 & 11 & 177.369886 \\
$\mathrm{C}$ & 14 & 2.773190 & 13 & 11.900673 & 12 & 173.121172 \\
$\mathrm{C}$ & 15 & 2.543467 & 14 & 23.303489 & 13 & 178.362170 \\
$\mathrm{~N}$ & 16 & 2.499798 & 15 & 176.589774 & 14 & -62.663866 \\
$\mathrm{C}$ & 17 & 1.374286 & 16 & 111.375791 & 15 & 48.872733 \\
$\mathrm{H}$ & 18 & 12.374475 & 17 & 58.985927 & 16 & -7.336387 \\
$\mathrm{C}$ & 19 & 1.091525 & 18 & 63.490918 & 17 & 171.078719 \\
$\mathrm{~F}$ & 20 & 14.326679 & 19 & 113.236146 & 18 & 0.611709 \\
$\mathrm{C}$ & 21 & 11.881641 & 20 & 2.305559 & 19 & 14.012068 \\
$\mathrm{C}$ & 22 & 2.540799 & 21 & 13.231919 & 20 & -173.611762 \\
$\mathrm{C}$ & 23 & 2.559394 & 22 & 179.665090 & 21 & -12.415728 \\
$\mathrm{H}$ & 24 & 5.241512 & 23 & 11.919240 & 22 & -165.814075 \\
$\mathrm{H}$ & 25 & 2.567510 & 24 & 12.283468 & 23 & -179.726790 \\
$\mathrm{H}$ & 26 & 2.580037 & 25 & 179.179260 & 24 & 63.344576 \\
$\mathrm{C}$ & 27 & 2.806035 & 26 & 154.591453 & 25 & -70.298787 \\
$\mathrm{H}$ & 28 & 1.094764 & 27 & 69.486078 & 26 & -176.311097 \\
$\mathrm{O}$ & 29 & 3.848383 & 28 & 20.472884 & 27 & 159.679660 \\
$\mathrm{C}$ & 30 & 12.152756 & 29 & 66.964198 & 28 & -23.987459 \\
$\mathrm{H}$ & 31 & 1.088750 & 30 & 69.475525 & 29 & -178.200041 \\
$\mathrm{H}$ & 32 & 3.390652 & 31 & 77.175321 & 30 & 8.254736 \\
$\mathrm{H}$ & 33 & 2.562516 & 32 & 155.239609 & 31 & -166.846622 \\
$\mathrm{H}$ & 34 & 2.533544 & 33 & 179.688564 & 32 & -141.919776 \\
$\mathrm{H}$ & 35 & 9.280758 & 34 & 1.484682 & 33 & 29.791253 \\
$\mathrm{H}$ & 36 & 11.789868 & 35 & 0.790609 & 34 & 115.684555
\end{tabular}

TFAAD anion $\quad E=\mathbf{- 8 9 8 . 7 4 9 1 4 7 6 9 1 ~ H a ~}$

F

$\mathrm{H} \quad 1 \quad 12.187614$ 


$\begin{array}{lllllll}\mathrm{H} & 2 & 2.541026 & 1 & 7.512578 & & \\ \mathrm{H} & 3 & 2.578146 & 2 & 179.462639 & 1 & 43.303555 \\ \mathrm{H} & 4 & 2.590984 & 3 & 179.470893 & 2 & -57.339307 \\ \mathrm{H} & 5 & 2.935806 & 4 & 172.201717 & 3 & -116.903693 \\ \mathrm{~F} & 6 & 3.866370 & 5 & 110.026447 & 4 & 109.609245 \\ \mathrm{C} & 7 & 1.399665 & 6 & 16.727745 & 5 & -146.017147 \\ \mathrm{C} & 8 & 12.030576 & 7 & 107.975161 & 6 & 38.916706 \\ \mathrm{H} & 9 & 5.278755 & 8 & 3.860857 & 7 & 24.962008 \\ \mathrm{H} & 10 & 2.586566 & 9 & 12.091702 & 8 & -164.147600 \\ \mathrm{C} & 11 & 1.101869 & 10 & 89.758775 & 9 & -1.784566 \\ \mathrm{H} & 12 & 2.828438 & 11 & 66.982112 & 10 & -179.924451 \\ \mathrm{H} & 13 & 7.792859 & 12 & 22.900726 & 11 & 0.769607 \\ \mathrm{C} & 14 & 2.830966 & 13 & 22.988765 & 12 & 0.316407 \\ \mathrm{C} & 15 & 2.579493 & 14 & 22.801347 & 13 & 179.627843 \\ \mathrm{~N} & 16 & 2.525997 & 15 & 177.240544 & 14 & -89.880649 \\ \mathrm{C} & 17 & 1.431254 & 16 & 107.037384 & 15 & 80.406832 \\ \mathrm{~F} & 18 & 2.690766 & 17 & 105.543813 & 16 & 179.487243 \\ \mathrm{C} & 19 & 14.738961 & 18 & 8.852804 & 17 & 26.280125 \\ \mathrm{H} & 20 & 1.094367 & 19 & 100.950472 & 18 & -163.982591 \\ \mathrm{C} & 21 & 2.860864 & 20 & 62.872728 & 19 & -2.236610 \\ \mathrm{H} & 22 & 1.102115 & 21 & 66.472967 & 20 & 178.656072 \\ \mathrm{C} & 23 & 2.810176 & 22 & 66.662373 & 21 & -178.946154 \\ \mathrm{C} & 24 & 2.583775 & 23 & 156.812983 & 22 & 179.581644 \\ \mathrm{H} & 25 & 2.812778 & 24 & 23.081112 & 23 & 1.371031 \\ \mathrm{C} & 26 & 5.282965 & 25 & 10.966933 & 24 & 178.803173 \\ \mathrm{H} & 27 & 2.810444 & 26 & 11.155883 & 25 & 179.057180 \\ \mathrm{H} & 28 & 2.604770 & 27 & 23.097530 & 26 & -179.131551 \\ \mathrm{C} & 29 & 11.474444 & 28 & 9.295804 & 27 & -12.616630 \\ \mathrm{H} & 30 & 1.089299 & 29 & 97.425049 & 28 & 168.099044 \\ \mathrm{O} & 31 & 12.294238 & 30 & 95.358024 & 29 & 2.266840 \\ \mathrm{H} & 32 & 13.393665 & 31 & 6.668966 & 30 & 16.554613 \\ \mathrm{H} & 33 & 4.438038 & 32 & 9.796902 & 31 & 173.106012 \\ \mathrm{H} & 34 & 2.594732 & 33 & 177.672552 & 32 & 86.967064 \\ \mathrm{H} & 35 & 2.582923 & 34 & 179.758097 & 33 & 20.473249 \\ \mathrm{H} & 36 & 2.541800 & 35 & 179.609903 & 34 & 139.127919\end{array}$




\section{References}

(1) Gabedit is a free Graphical User Interface for computational chemistry packages. It is written by Abdul-Rahman Allouche. Gabedit is available from http://lasim.univ-lyon1.fr/allouche/gabedit

(2) Sun, B.; Colavita, P. E.; Kim, H.; Lockett, M.; Marcus, M. S.; Smith, L. M.; Hamers, R. J. Langmuir 2006, 22, 9598-9605.

(3) Baker, S. E.; Colavita, P. E.; Tse, K. Y.; Hamers, R. J. Chem. Mater. 2006, 18, 4415-4422.

(4) Nuzzo, R. G.; Dubois, L. H.; Allara, D. L. J. Am. Chem. Soc. 1990, 112, 558-569.

(5) Porter, M. D.; Bright, T. B.; Allara, D. L.; Chidsey, C. E. D. J. Am. Chem. Soc. 1987, 109, 3559-3567.

(6) Haerle, R.; Riedo, E.; Pasquarello, A.; Baldereschi, A. Phys. Rev. B 2001, 65, 045101-9.

(7) Jackson, S. T.; Nuzzo, R. G. Appl. Surf. Sci. 1995, 90, 195-203.

(8) Poirier, D. M.; Weaver, J. H.; Kikuchi, K.; Achiba, Y. Z. Phys. D 1993, 26, 79-83.

(9) Atamny, F.; Blocker, J.; Dubotzky, A.; Kurt, H.; Timpe, O.; Loose, G.; Mahdi, W.; Schlogl, R. Mol. Phys. 1992, 76, 851-886.

(10) Kelemen, S. R.; RFreund, H.; Mims, C. A. J. Catal. 1986, 97, 228-239.

(11) Schlaf, R.; Schroeder, P. G.; Nelson, M. W.; Parkinson, B. A.; Lee, P. A.; Nebesny, K. W.; Armstrong, N. R. Journal of Applied Physics 1999, 86, 14991509.

(12) Ertl, G.; Küppers, J. Low Energy Electrons and Surface Chemistry; Wiley-VCH: Weinheim, 1986.

(13) Randles, J. E. B. Discuss. Faraday Soc. 1947, 1, 11-19.

(14) Bard, A. J.; Faulkner, L. Electrochemical Methods: Fundamentals and Applications; 2nd ed.; J. Wiley: New York, 2001.

(15) Gaussian 03, Revision C.02, Frisch, M. J.; Trucks, G. W.; Schlegel, H. B.; Scuseria, G. E.; Robb, M. A.; Cheeseman, J. R.; Jr., J. A. M.; Vreven, T.; Kudin, K. N.; Burant, J. C.; Millam, J. M.; Iyengar, S. S.; Tomasi, J.; Barone, V.; Mennucci, B.; Cossi, M.; Scalmani, G.; Rega, N.; Petersson, G. A.; Nakatsuji, H.; Hada, M.; Ehara, M.; Toyota, K.; Fukuda, R.; Hasegawa, J.; Ishida, M.; Nakajima, T.; Honda, Y.; Kitao, O.; Nakai, H.; Klene, M.; Li, X.; Knox, J. E.; Hratchian, H. 
P.; Cross, J. B.; Bakken, V.; Adamo, C.; Jaramillo, J.; Gomperts, R.; Stratmann, R. E.; Yazyev, O.; Austin, A. J.; Cammi, R.; Pomelli, C.; Ochterski, J. W.; Ayala, P. Y.; Morokuma, K.; Voth, G. A.; Salvador, P.; Dannenberg, J. J.; Zakrzewski, V. G.; Dapprich, S.; Daniels, A. D.; Strain, M. C.; Farkas, O.; Malick, D. K.; Rabuck, A. D.; Raghavachari, K.; Foresman, J. B.; Ortiz, J. V.; Cui, Q.; Baboul, A. G.; Clifford, S.; Cioslowski, J.; Stefanov, B. B.; Liu, G.; Liashenko, A.; Piskorz, P.; Komaromi, I.; Martin, R. L.; Fox, D. J.; Keith, T.; Al-Laham, M. A.; Peng, C. Y.; Nanayakkara, A.; Challacombe, M.; Gill, P. M. W.; Johnson, B.; Chen, W.; Wong, M. W.; Gonzalez, C.; Pople, J. A.; Gaussian, Inc.: Wallingford CT, 2004. 